\title{
Anéis vasculares na infância: diagnóstico e tratamento
}

\author{
Vascular rings in childhood: diagnosis and treatment
}

\author{
Luis R. Longo-Santos ${ }^{1}$, João G. Maksoud-Filho ${ }^{2}$, Uenis Tannuri ${ }^{3}$, Wagner C. Andrade ${ }^{4}$, \\ Manoel E.P. Gonçalves ${ }^{5}$, Silvia R. Cardoso ${ }^{5}$, João G. Maksoud ${ }^{6}$
}

\section{Resumo}

Objetivo: apresentar a experiência do Serviço de Cirurgia Pediátrica do Instituto da Criança do HCFMUSP no diagnóstico e tratamento de crianças com anomalias do arco aórtico e definir a importância dos exames complementares para o diagnóstico.

Método: estudo retrospectivo de 22 crianças com diagnóstico de compressão traqueoesofágica por anel vascular tratadas no Instituto da Criança, no período de 1985 a 2000, investigando-se dados clínicos pré e pós-operatórios, exames complementares e evolução.

Resultados: a anomalia vascular mais freqüente foi artéria inominada direita anômala (10 casos), seguido de duplo arco aórtico (7 casos) e arco aórtico à direita (5 casos). Os sintomas predominantes foram respiratórios $(86 \%)$ e de início precoce $(76 \%$ desde o período neonatal). Entretanto, o diagnóstico definitivo na maioria dos casos $(60 \%)$ só foi estabelecido após 1 ano de vida. O exame mais importante para o diagnóstico foi o esofagograma. A correção de todas anomalias foi realizada por toracotomia póstero-lateral esquerda. Não ocorreram complicações cirúrgicas. A evolução foi pior nos casos operados mais tardiamente. Todas as crianças permaneceram sintomáticas por até 6 meses, apesar de significativa melhora no pós-operatório.

Conclusão: o diagnóstico de anel vascular deve ser investigado nas crianças com sintomas respiratórios de início precoce e nas "chiadoras" de difícil controle. O diagnóstico pode ser realizado de forma simples através do esofagograma. Os demais exames de imagem acrescentam poucas informações e são dispensáveis na maioria dos casos. Os sintomas respiratórios podem persistir com menor intensidade por períodos variáveis no pós-operatório.

J Pediatr (Rio J) 2002; 78 (3): 244-50: obstrução das vias respiratórias, estenose traqueal, bebê chiador, estenose esofágica, disfagia lusória, anomalia da aorta torácica, cirurgia da aorta torácica.

\begin{abstract}
Objective: To present the study carried out by the Pediatric Surgery Department of Instituto da Criança at the Medical School of Universidade de São Paulo regarding the diagnosis and treatment of children with aortic arch abnormalities and to define the role of complementary exams for diagnosis.
\end{abstract}

Methods: Retrospective study of 22 patients with diagnosis of tracheoesophageal compression treated at Instituto da Criança from 1985 to 2000, analyzing pre- and postoperative clinical data, diagnostic exams and outcome.

Results: The most frequent diagnosis was right aberrant innominate artery (10 cases), followed by double aortic arch ( 7 cases) and right aortic arch (5 cases). Respiratory symptoms (86\%) and early manifestation ( $76 \%$ since the neonatal period) were predominant. Nevertheless, most cases $(60 \%)$ had the definitive diagnosis established only after 1 year of life. The most relevant examination for the diagnosis was the esophagogram. The correction of all the anomalies was carried out through left postero-lateral thoracotomy. There were no surgical complications. The outcome was worse in patients with delayed treatment. All children remained symptomatic for up to 6 months, although they had significant improvement in the postoperative period.

Conclusions: The diagnosis of vascular rings should be considered in children with early respiratory symptoms and in the wheezing baby with difficult control. The diagnosis may be established just through the esophagogram. Other image studies add few information and they are unnecessary in most cases. Less severe symptoms may persist for variable periods.

J Pediatr (Rio J) 2002; 78 (3):244-50: airway obstruction, tracheal stenosis, wheezing baby, esophageal stenosis, dysphagia lusoria, abnormalities of the thoracic aorta, surgery of the thoracic aorta.

1. Médico preceptor - Disciplina de Cirurgia Pediátrica - HCFMUSP.

2. Doutor em Cirurgia. Médico do Serviço de Cirurgia Pediátrica do ICr-HCFMUSP.

3. Prof. Associado - Disciplina de Cirurgia Pediátrica - FMUSP.

4. Residente de Cirurgia Pediátrica do ICr-HCFMUSP.

5. Endoscopista do ICr-HCFMUSP.

6. Prof. Titular - Disciplina de Cirurgia Pediátrica - FMUSP.

Artigo submetido em 04.10.01, aceito em 27.03.02. 


\section{Introdução}

As anomalias congênitas do arco aórtico, também conhecidas como anéis vasculares e anomalias dos vasos da base, constituem um grupo de malformações que causam compressão do esôfago e/ou da traquéia, sendo responsáveis por sintomas respiratórios e digestivos. São malformações raras, classicamente subdivididas em anéis completos, como o duplo arco aórtico (DAA) e o arco aórtico à direita, com persistência do ducto arterioso (AD/LE), e em anéis incompletos, como artéria subclávia direita anômala (AI) e anel de artéria pulmonar ${ }^{1,2}$.

As malformações do arco aórtico foram descritas inicialmente em adultos, os quais apresentavam sintomas predominantemente digestivos. Em conseqüência disso, nos relatos clássicos encontra-se a denominação "disfagia lusória" para nomear a compressão vascular do esôfago ${ }^{3}$. Entretanto a experiência em pediatria mostra que o início dos sintomas ocorre freqüentemente nos primeiros meses de vida, e que esses são predominantemente relacionados ao sistema respiratório ${ }^{4-7}$.

Embora o diagnóstico genérico de compressão traqueal e/ou esofágica possa ser estabelecido de maneira simples, ele é, na maioria das vezes, definido tardiamente, retardando o tratamento cirúrgico. Tal fato pode ser atribuido à diversidade dos sintomas, que, muitas vezes, são confundidos com afecções pulmonares ou com refluxo gastroesofágico $^{8}$. O desconhecimento acerca do diagnóstico de anel vascular e, conseqüentemente, o baixo índice de suspeita são outros fatores importantes para o atraso no tratamento definitivo.

Vários autores ressaltaram a importância deste diagnóstico diferencial na investigação de crianças com sintomas respiratórios crônicos (bebês chiadores) e discutiram a seqüência propedêutica adequada em suas experiências ${ }^{4-}$ 6,8-10. A utilização de novos exames de imagem não acrescentou necessariamente vantagens para a investigação.

O objetivo do presente trabalho é mostrar a experiência do Serviço de Cirurgia Pediátrica do Instituto da Criança do Hospital das Clínicas da Faculdade de Medicina da USP (ICr-HCFMUSP) no diagnóstico e tratamento das anomalias do arco aórtico, e definir o papel dos diversos exames complementares no diagnóstico dessas malformações ${ }^{9-11}$.

\section{Métodos}

De fevereiro de 1985 a abril de 2000, 22 crianças portadoras de anel vascular foram admitidas no Serviço de Cirurgia Pediátrica do Instituto da Criança. Os tipos de anel vascular foram classificados conforme nomenclatura estabelecida pelo Congenital Heart Surgery Database Commitee e pelo European Association for Cardiothoracic Surgery $^{1}$ (Tabela 1).

Os dados clínicos pré e pós-operatórios, os procedimentos diagnósticos e a evolução pós-operatória imediata e tardia foram obtidos por análise retrospectiva dos prontuários.
Tabela 1 - Incidência dos anéis vasculares

\begin{tabular}{lcc}
\hline Tipo & N & \% casos \\
\hline AI & 10 & $45 \%$ \\
DAA & 7 & $32 \%$ \\
AD/LE & 5 & $23 \%$ \\
Total & 22 & $100 \%$ \\
\hline
\end{tabular}

Al: compressão inominada (artéria subclávia direita anômala). DAA: duplo arco aórtico.

AD/LE: arco aórtico à direita/ligamento arterioso à esquerda.

\section{Resultados \\ Sintomas pré-operatórios}

Em 16 crianças (73\%), os sintomas surgiram no período neonatal, sendo que duas crianças apresentaram insuficiência respiratória aguda, necessitando de intubação orotraqueal após o nascimento. Nas demais, o início dos sintomas ocorreu entre o $2^{\circ}$ e o $6^{\circ}$ mês de vida.

Os sintomas respiratórios predominaram, sendo mais freqüentes crises de broncoespasmo ou chiado perene, presentes em 19 pacientes (86\%). Essas crianças foram inicialmente tratadas como "bebê chiador", sem melhora até que o diagnóstico correto fosse estabelecido. Outros sintomas respiratórios, como estridor respiratório e pneumonias de repetição, foram relatados, assim como sintomas digestivos (Tabela 2). Estes últimos foram os menos freqüentes e pouco significativos, nunca constituindo a queixa principal.

Tabela 2 - Sintomas predominantes

\begin{tabular}{lcc}
\hline Sintomas & $\mathbf{N}$ & \% casos \\
\hline Chiador crônico & 19 & $86 \%$ \\
Cianose às mamadas & 10 & $45 \%$ \\
Estridor & 9 & $41 \%$ \\
Disfagia/engasgo & 8 & $36 \%$ \\
Total & 22 & $100 \%$ \\
\hline
\end{tabular}

Quatorze crianças (63\%) apresentaram mais de um episódio de pneumonia antes do diagnóstico definitivo, cinco das quais chegaram a necessitar de ventilação mecânica e internação em terapia intensiva.

\section{Diagnóstico}

Apesar dos sintomas precoces, na maioria dos casos (60\%) o diagnóstico de compressão por anel vascular somente foi estabelecido em idade superior a 1 ano. 
Com exceção de dois pacientes, todos os demais foram submetidos à esofagograma com bário, sendo possível identificar compressão em terço médio do esôfago e classificar o provável tipo de malformação.

A traqueobroncoscopia foi realizada em 15 crianças (68\%), revelando genericamente compressão pulsátil no terço inferior da traquéia. Dois recém-nascidos submetidos à ventilação mecânica devido à insuficiência respiratória aguda grave tiveram o diagnóstico firmado exclusivamente por traqueobroncoscopia, em função da impossibilidade de realização do esofagograma. A traqueobroncoscopia também permitiu o diagnóstico de traqueomalácia grave associada em três pacientes (14\%).

Em dez crianças, foram realizados outros exames de imagem; em nove delas, realizou-se tomografia computadorizada de tórax; em uma, ressonância nuclear magnética, e em três, arteriografia. Esses exames apenas confirmaram o diagnóstico efetivamente já firmado através do esofagograma. Nenhum deles modificou o diagnóstico inicial, a indicação ou a abordagem cirúrgica.

Em sete crianças, realizou-se ecocardiograma, com o objetivo de investigar eventuais malformações cardíacas associadas. Três apresentavam sopro cardíaco, e nelas foram diagnosticadas comunicação interatrial (2 casos) e comunicação interventricular (1 caso). Em nenhum dos sete casos o ecocardiograma conseguiu identificar o anel vascular.

\section{Cirurgia}

A idade das crianças por ocasião da cirurgia variou de 21 dias a 4 anos e 4 meses.

A via de acesso foi em todos os casos toracotomia póstero-lateral esquerda transpleural ${ }^{12,13}$.

Nos casos de duplo arco aórtico (DAA), realizou-se a ligadura do arco anterior/esquerdo, que era o não dominante, seguida de ligadura do ducto arterioso fibrosado.

Nos casos de arco aórtico à direita (AD/LE), realizaramse a secção do ducto arterioso fibrosado e a liberação das aderências mediastinais, com o objetivo de aliviar a compressão esofágica e traqueal.

Nos casos de artéria inominada anômala (AI), realizou-se a ligadura e secção da artéria, sem necessidade de reimplante arterial. $\mathrm{O}$ fluxo no membro superior direito manteve-se normal, monitorado com oxímetro de pulso.

Uma criança portadora de AD/LE foi reoperada 6 dias após a primeira cirurgia, com indicação baseada na manutenção de sintomas intensos e de compressão traqueal à broncoscopia. Após dissecção ampla, com liberação de aderências mediastinais, obteve-se melhora significativa.

\section{Pós-operatório}

Exceto duas crianças, a que necessitou de reoperação e outra portadora de traqueomalácia grave, as demais tiveram significativa melhora dos sintomas a partir do pós-operatório imediato. Entretanto, em nenhum caso os sintomas se resolveram completamente no período inicial, persistindo por até 6 meses após a cirurgia.

Não houve complicações intra ou pós-operatórias imediatas. Três crianças faleceram no pós-operatório tardio, duas delas devido a complicações decorrentes de pneumopatia crônica e infecções de repetição, aos 6 meses e dois anos após a cirurgia, respectivamente. Essas crianças foram as primeiras desta série, e tiveram diagnóstico mais tardio, ou seja, com mais de 4 anos de idade. O exame anatomopatológico dos pulmões nesses casos revelou pneumopatia crônica com sinais de bronquiolite obliterante. A terceira criança faleceu em decorrência de complicações de traqueomalácia grave.

\section{Discussão}

Embora raras, as malformações do arco aórtico são facilmente diagnosticadas, com investigação simples a partir de manifestação clínica inespecífica, porém significativa. Em nossa casuística, todas as crianças apresentavam sintomas respiratórios, a maioria de início logo após o nascimento. Crianças com sintomas de obstrução respiratória alta (cianose às mamadas, estridor inspiratório) ou asma de difícil controle devem ser precocemente investigadas, com o objetivo de identificar a presença de compressão traqueal, fazendo parte da preocupação inicial de todo pediatra que acompanha um "bebê chiador".

Como diagnósticos diferenciais adicionais, podemos acrescentar outras massas torácicas que podem causar compressão do esôfago e traquéia, como pequenos cistos broncogênicos, cistos de duplicação esofágico e tumores benignos e malignos do mediastino. Nesses casos, os achados radiológico e endoscópico não-característicos de anel vascular podem indicar investigação complementar, embora a conduta permaneça cirúrgica.

A traqueobroncoscopia é exame objetivo e muito útil para o diagnóstico destas malformações ${ }^{14}$. Em alguns casos, principalmente naqueles com sintomas respiratórios intensos, foi o primeiro exame a ser solicitado. Também em crianças intubadas devido à insuficiência respiratória grave, a traqueobroncoscopia foi essencial no diagnóstico, pela impossibilidade de realização do esofagograma.

A tomografia computadorizada (TC) e a ressonância nuclear magnética (RNM), embora freqüentemente solicitadas, em nada contribuem para o diagnóstico. Exceto quando indicadas com objetivo específico de definir outras formas de compressão extrínseca da traquéia e do esôfago, como cistos e tumores mediastinais, não acrescentam informações relevantes para o diagnóstico das anomalias do arco aórtico. Além disso, na maioria dos casos de anel vascular, os vasos anômalos não são pérvi- 
os, e, portanto, não podem ser contrastados, dificultando a interpretação das imagens ${ }^{10,11,15}$.

A arteriografia é exame invasivo, e deve ser indicado especificamente nos casos de cardiopatia complexa, ou quando se suspeita de anel da artéria pulmonar (casos com compressão da parede anterior do esôfago e do brônquio E). Nas demais situações, a arteriografia pode ser evitada, visto que a definição precisa da malformação é feita no próprio ato operatório e, como já referido, em muitas situações as estruturas do anel vascular são atrésicas, e não são contrastadas na arteriografia.

O ecocardiograma é útil para investigação de malformações cardíacas associadas ${ }^{16}$, mas não permite o diagnóstico diferencial dos anéis vasculares. Nesta casuística, as malformações cardíacas foram pouco freqüentes $(13,6 \%)$, sem repercussão clínica, e não necessitaram de correção cirúrgica concomitante.

O esofagograma é, juntamente com o exame endoscópico, o principal método diagnóstico ${ }^{17}$. Apenas com o esofagograma foi possível identificar a existência de compressão no terço médio do esôfago em 20 casos, sendo suficiente para diagnosticar com precisão o tipo de malformação em 18 deles $(90 \%)$, conforme a imagem obtida. Em apenas dois casos o tipo de malformação sugerido pelo esofagograma não coincidiu com o achado intra-operatório (esofagograma interpretado como de DAA com diagnóstico intraoperatório de $\mathrm{AD} / \mathrm{LE}$ e diagnóstico pré-operatório de $\mathrm{AI}$ em criança com DAA). Entretanto, o engano na definição diagnóstica pré-operatória não levou a qualquer prejuízo para o tratamento cirúrgico, pois através do mesmo acesso cirúrgico foi definida a malformação e realizada a correção adequada. As imagens características ${ }^{17}$ correspondentes a cada tipo de anel vascular são descritas nas Figuras 1, 2 e 3.

Em relação ao tratamento cirúrgico, em todos os casos foi realizada a ligadura e secção de estruturas que permitissem liberar plenamente a compressão esofágica e traqueal sem prejudicar o fluxo vascular. Assim, através de ligadura do ducto arterioso e do arco aórtico atrésico ou nãodominante, é possível resolver os casos de DAA e de AD/LE. Nos casos de AI, embora alguns autores indiquem reimplante da artéria subclávia direita em sua posição anatômica $^{18}$, em nossa experiência esse procedimento é dispensável e acrescentaria morbidade cirúrgica desnecessária, visto que o fluxo distal se restabelece imediatamente após o clampeamento da artéria subclávia a partir de colaterais, sem prejuízo funcional. $\mathrm{O}$ acesso cirúrgico através de toracotomia póstero-lateral esquerda permite excelente exposição das estruturas vasculares e mediastinais, possibilitando a definição da anatomia da anomalia e da tática cirúrgica adequada para sua correção. Permite, também, a liberação de aderências fibrosas que podem persistir após a ligadura vascular, evitando manutenção de sintomas compressivos. Com exceção do anel da artéria pulmonar, anomalia muito rara e diagnosticada quando a compressão é identificada na parede anterior do esôfago, nas demais anomalias do arco aórtico não é necessária circulação extracorpórea para correção.

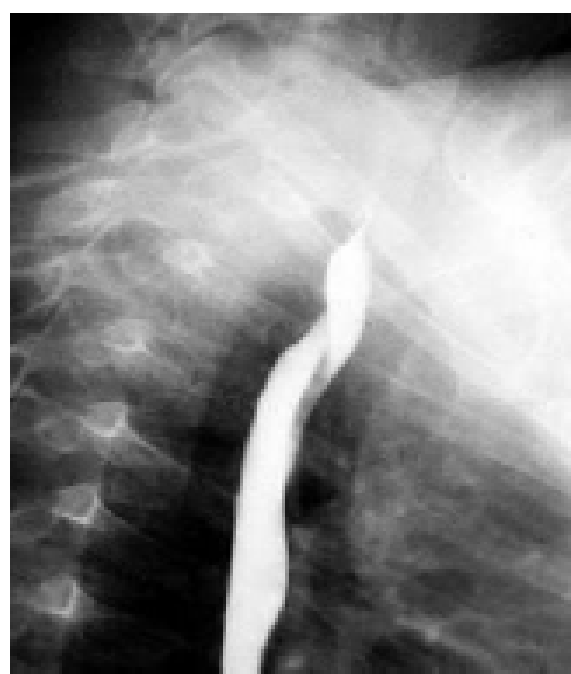

A

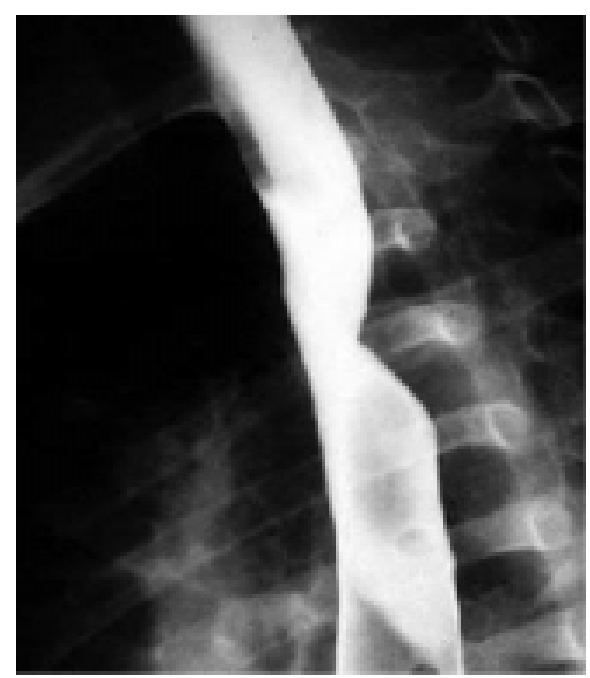

B

Figura 1 - Esofagograma de artéria subclávia direita anômala. A - radiografia em oblíquo; B -radiografia em perfil. Impressão oblíqua e posterior, melhor visualizada como uma compressão aguda posterior na radiografia em perfil 


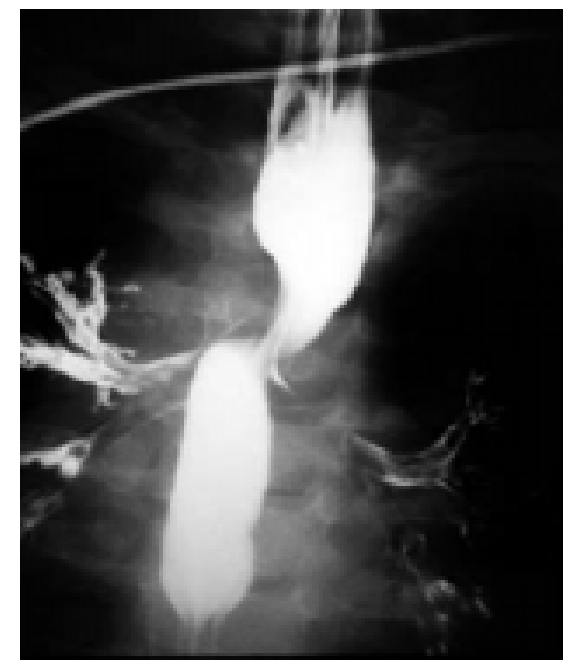

A

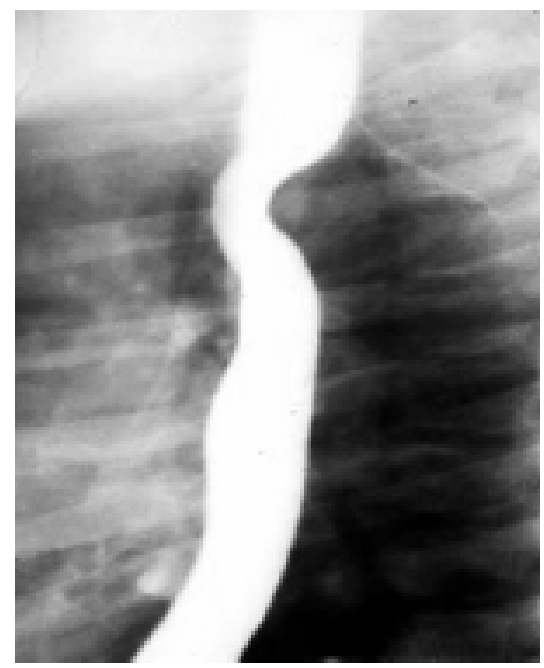

B

Figura 2 - Esofagograma de duplo arco aórtico. A - radiografia em AP; B - radiografia em perfil. Duas compressões esofágicas: uma maior, superior e à direita, que corresponde ao arco aórtico direito, e outra menor, inferior e à esquerda, que corresponde ao arco aórtico esquerdo. Em perfil, observa-se compressão grande e arredondada na parede posterior do esôfago, correspondente ao arco aórtico direito

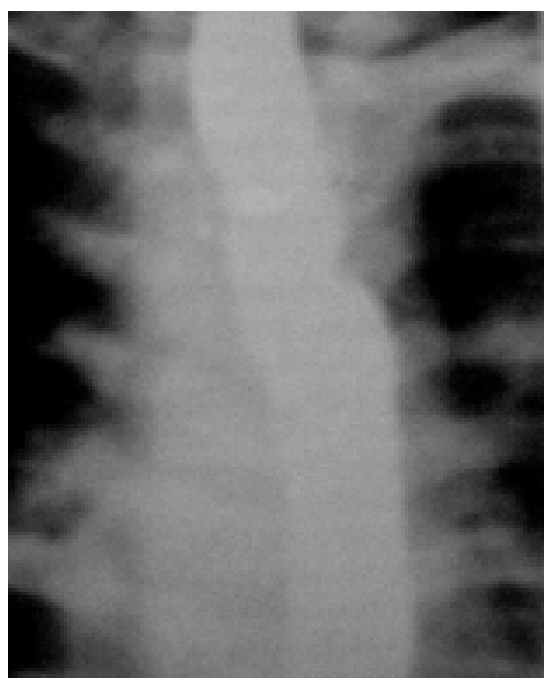

A

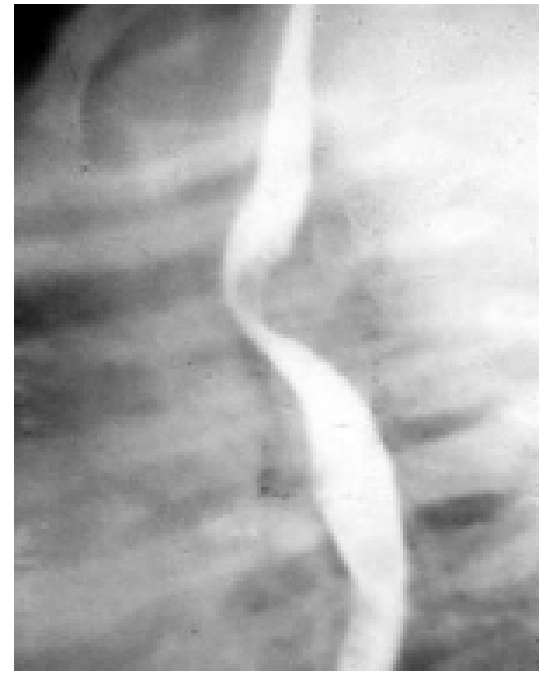

B

Figura 3 - Esofagograma de arco aórtico à D com ligamento arterioso à E. A - radiografia em PA; B - radiografia em perfil. Compressão esofagiana discreta superior à direita, correspondente ao arco aórtico $\mathrm{D}$, e outra inferior à esquerda, correspondente à artéria subclávia E. Em perfil, compressão posterior pronunciada, correspondente ao bulbo de origem da artéria subclávia $\mathrm{E}$ 
A incidência de traqueomalácia associada aos anéis vasculares foi de cerca de $14 \%$. A traqueomalácia e as pneumopatias crônicas são as causas mais freqüentes da manutenção de sintomas respiratórios no pós-operatório. Esses sintomas residuais podem ser relacionados a alterações na dinâmica traqueal, lesões crônicas do parênquima pulmonar, hiper-reatividade crônica de vias aéreas e infecções de repetição ${ }^{7,19,20}$. No pós-operatório, a fisioterapia respiratória e a antibioticoprofilaxia são de grande valor no tratamento a longo prazo dessas crianças.

\section{Referências bibliográficas}

1. Backer CL, Mavroudis C. Congenital heart surgery nomenclature and database project: vascular rings, tracheal stenosis, pectus excavatum. Ann Thorac Surg 2000;64-4 Suppl:382-18.

2. Maksoud-Filho JG, Maksoud JG. Anomalias do arco aórtico. In: Makosud JG. Cirurgia Pediátrica. $1^{a}$ ed. Rio de Janeiro: Revinter; 1998.p574-82.

3. Palmer ED. Disfagia lusoria: clinical aspects in adults. Ann Int Med 1955; 42:1173-79.

4. Kocis KC, Midgley FM, Ruckman RN. Aortic arch complex anomalies: 20-year experience with symptoms, diagnosis, associated cardiac defects, and surgical repair. Pediatr Cardiol 1997; 18:127-32.

5. Krzystolik-Ladzinska J, Wiecek-Wlodarska D, Guzikowski K, Rokicki W, Wites M, Pieniazek P. Vascular rings as a cause of the respiratory disturbances in children. Wiad Lek 2000; 53:289-98.

6. Backer CL, Ilbawi MN, De Leon SY. Vascular anomalies causing tracheoesophageal compression. Review of the experience in children. J Thorac Cardiovasc Surg 1989;97:725-30.

7. Thomson AH, Beardsmore CS, Firmin R, Leanage R, Simpson $\mathrm{H}$. Airway function in infants with vascular rings: preoperative and posoperative assessment. Arch Dis Child 1990; 65:171-4.

8. Bakker DA, Berger RM, Borges AJ. Vascular rings: a rare cause for common respiratory symptoms. Acta Paediatr 1999; 88:947-52.

9. Sarbeji M, De Blic J, Le Baugeois M, Mamou MT, Revillon Y, Paupe J. Practical diagnostic approach to anomalies of the aortic arch. A propos of 31 pediatric cases. Rev Mal Resp 1990;7:51-7.
A análise dessa casuística permite-nos concluir que o diagnóstico de anel vascular deve ser suspeitado em crianças com sintomas respiratórios iniciados no período neonatal, e em crianças com diagnóstico genérico de "bebê chiador" de difícil controle. O diagnóstico pode ser feito de forma simples, através do esofagograma e endoscopia traqueal e digestiva. Os demais exames de imagem acrescentam poucas informações e são dispensáveis. Finalmente, os sintomas respiratórios podem permanecer no pósoperatório por períodos variáveis, em decorrência de traqueomalácia ou afecções pulmonares crônicas.
10. Chunk K, Colombani PM, Dudgeon DL, Haller JA. Diagnosis and management of congenital vascular rings: a 22-year experience. Ann Thorac Surg 1992;53:597-602.

11. van Son JA, Julsrud PR, Hagler DJ, Pugo FJ, Schaff HV, Danielson GK, et al. Imaging strategies for vascular rings. Ann Thorac Surg 1994; 57:604-10.

12. Backer CL, Mavroudis C. Surgical approach to vascular rings. Adv Card Surg 1997; 9:29-64.

13. Richardson JV, Doty DB, Rossi NP, Ehrenhaft JL. Operations for aortic arch anomalies. Ann Thorac Surg 1981; 31:426-32.

14. Chapotte C, Monrigal JP, Pezard P, Jeudy C, Subayi JB,Granry JC, et al. Airway compression in children due to congenital heart disease: value of flexible fiberoptic bronchoscopic assessment. J Cardiothorac Vasc Anesth 1998; 12:145-52.

15. Maksoud-Filho JG, Gonçalves MEP, Tannuri U, Maksoud JG. Compressões do esôfago e da traquéia por anomalias do arco aórtico na infância. Rev Ass Med Brasil 1993; 39:155-9.

16. Burch M, Deanfield JE, Sullivan ED. Investigation of vascular compressions of trachea: the complementary roles of barium swalow and echocardiography. Arch Dis Child 1993; 68:171-5.

17. Neuhauser BD. The roentgen diagnosis of double aaortic arch and other anomalies of great vessels. Am J Roengen Radiumther 1946; 56:188-91.

18. Hawkins JA, Baily WW, Clark JM. Innominate artery compression of trachea. Treatment by reimplantation of the innominate artery. J Thorac Cardiovasc Surg 1992; 103:678-82. 
19. Bertrand JM, Chartrand C, hamarre A, Lapierre JG. Vascular ring: clinical and physiological assessment of pulmonary function following surgical correction. Pediatr Pulmonol 1986; 2:378-83.

20. Marmon LM, Bye MR, Haas JM, Balsara RK, Dunn JM. Vascular rings and slings: long term follow up of pulmonary functions. J Pediatr Surg 1984; 19:683-92.
Endereço para correspondência:

Dr. Luis Ricardo Longo dos Santos

Rua Artur Bernardes, 265 - Jd. Sta. Francisca

CEP 07013-030 - Guarulhos, SP

E-mail:luisrls@hcnet.usp.br 